\title{
INFINITE GROUPS WITH LARGE BALLS OF TORSION ELEMENTS AND SMALL ENTROPY
}

\author{
LAURENT BARTHOLDI AND YVES DE CORNULIER
}

\begin{abstract}
We exhibit infinite, solvable, virtually abelian groups with a fixed number of generators, having arbitrarily large balls consisting of torsion elements. We also provide a sequence of 3-generator non-virtually nilpotent polycyclic groups of algebraic entropy tending to zero. All these examples are obtained by taking appropriate quotients of finitely presented groups mapping onto the first Grigorchuk group.
\end{abstract}

The Burnside Problem asks whether a finitely generated group all of whose elements have finite order must be finite. We are interested in the following related question: fix $n$ sufficiently large; given a group $\Gamma$, with a finite symmetric generating subset $S$ such that every element in the $n$-ball is torsion, is $\Gamma$ finite? Since the Burnside problem has a negative answer, a fortiori the answer to our question is negative in general. However, it is natural to ask for it in some classes of finitely generated groups for which the Burnside Problem has a positive answer, such as linear groups or solvable groups. This motivates the following proposition, which in particular answers a question of Breuillard to the authors.

Proposition 1. For every n, there exists a group $G$, generated by a 3-element subset $S$ consisting of elements of order 2 , in which the $n$-ball consists of torsion elements, and which satisfies one of the additional assumptions:

(i) $G$ is solvable, virtually abelian, and infinite (more precisely, it has a free abelian normal subgroup of finite 2-power index); in particular it is linear.

(ii) $G$ is polycyclic but not virtually nilpotent.

(iii) $G$ is solvable but not polycyclic.

Remark 2.

(1) The groups in Proposition 1 can actually be chosen to be 2-generated: indeed, if $G$ is generated by three involutions $a, b, c$, then the subgroup generated by $a b$ and $b c$ has index at most two.

(2) Natural stronger hypotheses are the following: being linear in fixed dimension; being solvable of given solvability length. We have no answer in these cases. It is also natural to ask what happens it we fix a torsion exponent.

(3) By [Se, Corollaire 2, p. 90], if $G$ is a group and $S$ is any finite generating subset for which the 2-ball of $G$ consists of torsion elements, then $G$ has Property (FA): every action of $G$ on a tree has a fixed point. In particular, if $G$ is infinite, then by Stallings' Theorem St it cannot be virtually free.

(4) For every sufficiently large prime $p$, and for all $n$, there exists a non-elementary, 2 -generated word hyperbolic group in which the $n$-ball consists of elements of $p$-torsion [Ol].

Date: October 7, 2005.

2000 Mathematics Subject Classification. Primary 20F50; Secondary 20F16, 20 F05.

Key words and phrases. Grigorchuk group; balls of torsion; algebraic entropy. 
(5) We give more precise statements in the sequel: in (i), the free abelian subgroup can be chosen of index $2^{a_{n}}$, where $a_{n} \sim 13 n^{k}$ (that is, $a_{n} /\left(13 n^{k}\right) \rightarrow 1$ ), where $k \cong 6.60$ is a constant (see Corollary 10).

With a similar construction, we obtain results on the growth exponent. Let $G$ be generated by a finite symmetric set $S$, and denote by $B_{n}$ the $n$-ball in $G$. Then, by a standard argument [H], Proposition VI.56], the limit $h(G, S)=\lim \frac{1}{n} \log \left(\#\left(B_{n}\right)\right)$ exists. The (algebraic) entropy of $G$ is defined as $h(G)=\inf _{S} h(G, S)$, where $S$ ranges over all finite symmetric generating subsets of $G$. Osin has proved [Os1, Os2] that, for an elementary amenable finitely generated group, $h(G)=0$ if and only if $G$ is virtually nilpotent; on the other hand, Wilson [W] has constructed a finitely generated group with $h(G)=0$ which is not virtually nilpotent; see [B2] for a simpler example. Relying on former work of Grigorchuk G2, Osin observes in Os2 that there exist elementary amenable groups (actually they are virtually solvable) with $h>0$ arbitrary close to 0 . This last result can be improved as follows.

Proposition 3. For every $\varepsilon>0$, there exists a polycyclic, virtually metabelian, 3-generated group $G$ with $0<h(G)<\varepsilon$.

Propositions 1 and 3 are obtained by approximating the Grigorchuk group, first introduced in G1], by finitely presented groups.

We recall below the definition of a family of 3 -generated groups $\Gamma_{n}$, which are successive quotients $\left(\Gamma_{n+1}\right.$ is a quotient of $\Gamma_{n}$ for all $\left.n\right)$. These are finitely presented groups obtained by truncating a presentation of Grigorchuk's group. They are proved in [GH] to be virtually direct products of nonabelian free groups; they have larger and larger balls of torsion elements, and their entropy tends to zero. We get Propositions [1] and [3 by considering appropriate solvable quotients of the groups $\Gamma_{n}$.

Following Lysionok $\mathrm{L}$, the first Grigorchuk group is presented as follows. We start from the 3-generated group $\Gamma_{-1}=\left\langle a, b, c, d \mid a^{2}=b^{2}=c^{2}=d^{2}=b c d=1\right\rangle$. Elements $u_{n}$ and $v_{n}$ of $\Gamma_{-1}$ are defined below. Then, for $0 \leq n \leq \infty, \Gamma_{n}$ is defined as the quotient of $\Gamma_{-1}$ by the relations $u_{i}$ for $i<n+1$ and $v_{i}$ for $i<n$. The first Grigorchuk group $\Gamma=\Gamma_{\infty}$ has a wealth of remarkable properties. The most celebrated one is that $\Gamma$ has non-polynomial subexponential growth G2. It is also a 2-group, i.e. a group in which every element is of finite 2-power order, and is just-infinite, i.e. it is infinite but all its proper quotients are finite.

We now construct the relations $u_{n}$ and $v_{n}$. Consider the substitution $\sigma$ defined by $\sigma(a)=a c a, \sigma(b)=d, \sigma(c)=b, \sigma(d)=c$; extend its definition to words in the natural way, and finally observe that it defines a group endomorphism of $\Gamma_{-1}$. Set $u_{0}=(a d)^{4}, v_{0}=(\text { adacac })^{4}, u_{n}=\sigma^{n}\left(u_{0}\right), v_{n}=\sigma^{n}\left(v_{0}\right)$.

For all $n \geq-1$, the natural homomorphism $\Gamma_{n} \rightarrow \mathbf{Z} / 2 \mathbf{Z}$ sending $b, c, d$ to 0 and $a$ to 1 has kernel $\Xi_{n}$ of index two.

We will focus on the finitely presented groups $\Gamma_{n}$ rather than on $\Gamma$. Individually, and up to commensurability, the structure of these groups is not of special interest: $\Gamma_{n}$ is commensurable to a direct product of $2^{n}$ non-abelian free groups GH, Proposition 12]. However, since $\Gamma_{\infty}$ is torsion, for all $n$, there exists $i(n)$ such that every element in the $n$-ball of $\Gamma_{i(n)}$ is torsion. A quantitative statement is given in the following proposition, whose proof appears in the appendix. Let $\lambda \cong 1.25$ be the real root of the polynomial $2 X^{3}-X^{2}-X-1$, and set $i(n)=\left\lfloor\log _{\lambda}(n)-1\right\rfloor$. 
Proposition 4. In the $n$-ball of $\Gamma_{i(n)}$ (for the word metric), every element is of $2^{i(n)+1}$-torsion.

The following proposition, which specifies [GH, Proposition 12], describes the structure of $\Gamma_{n}$.

Proposition 5. For every $n \geq 0, \Gamma_{n}$ has a normal subgroup $H_{n}$ of index $2^{\alpha_{n}}$, where $\alpha_{n} \leq\left(13 \cdot 4^{n}-1\right) / 3$, and $H_{n}$ is a subgroup of index $2^{\beta_{n}}$ in a finite direct product of $2^{n}$ nonabelian free groups of rank 3 , where $\beta_{n} \leq\left(13 \cdot 4^{n}-15 \cdot 2^{n}+2\right) / 3$.

Remark 6. The main difference with GH] Proposition 12] is that the finite index subgroup they construct is not normal. Of course one could take a smaller normal subgroup of finite index, but this one need not a priori be of 2-power index, a fact we require to obtain solvable (and not only virtually solvable) groups in Propositions 1 and 3 .

We use the following elementary lemma.

Lemma 7. Let $G$ be a group, and let $H$ be a proper subgroup of index $2^{a}$ in $G$, normalized by a subgroup of index two in $G$. Let $N$ be the intersection of all conjugates of $H$. Then $N$ has index $2^{b}$ in $G$, for some integer $b \leq 2 a-1$.

Proof. If $H$ is normal in $G$, the result is trivial. Otherwise, consider the unique conjugate $H^{\prime} \neq H$ of $H$, so that $N=H \cap H^{\prime}$. Taking the quotient by $N$, we can suppose that $H \cap H^{\prime}=\{1\}$ and we are reduced to proving that $G$ is a 2-group of order $d \leq 2^{2 a-1}$. Let $W$ be the normalizer of $H$. Since it has index 2 in $G$, it is normal in $G$, so that $H^{\prime} \subset W$. Since $H$ and $H^{\prime}$ are both normal subgroups of $W$ and $H \cap H^{\prime}=\{1\},\left[H, H^{\prime}\right]=\{1\}$. Accordingly, $H H^{\prime}$ is a normal subgroup of $G$, contained in $W$, and is naturally the direct product of $H$ and $H^{\prime}$. The order of $H$ is $d / 2^{a}$, so that the order of $H H^{\prime}$ is $d^{2} / 2^{2 a}$, and hence the index of $H H^{\prime}$ in $G$ is $2^{2 a} / d$. This proves that $d$ is a power of 2 , and $d \leq 2^{2 a}$; actually $d \leq 2^{2 a-1}$ because $H H^{\prime}$ is contained in $W$, hence has index $\geq 2$ in $G$.

Remark 8. In Lemma 7. the assumption that the normalizer has index at most two is sharp: in the alternating group $A_{4}$, there are four subgroups of index 4 , all conjugate; they have pairwise trivial intersection, hence of index 12 , which is not a power of 2 .

Recall that $\Xi_{0} \subset \Gamma_{0}$ is a subgroup of index 2; it is generated by $b, c, d, a b a$, $a c a$, ada. By [GH, Proposition 1], the assignment $i_{0}(b)=(a, c), i_{0}(c)=(a, d)$, $i_{0}(d)=(1, b)$ extends to a unique group homomorphism $i_{0}: \Xi_{0} \rightarrow \Gamma_{-1} \times \Gamma_{-1}$ such that, for all $x \in \Gamma_{0}$, if $i_{0}(x)=\left(x_{0}, x_{1}\right)$, then $i_{0}(a x a)=\left(x_{1}, x_{0}\right)$. By [GH] Proposition 10], this induces, for all $n \geq 1$, an injective group homomorphism: $i_{n}: \Xi_{n} \rightarrow \Gamma_{n-1} \times \Gamma_{n-1}$.

Proof of Proposition 囵. Let us proceed by induction on $n$. We start with the essential case when $n=0$, worked out in [GH, Lemma 11]. Write $\Gamma_{0}=\langle a, b, d| a^{2}=b^{2}=$ $\left.d^{2}=(b d)^{2}=(a d)^{4}=1\right\rangle$ (this is a Coxeter group). Let $H_{0}$ be the normal subgroup generated by $(a b)^{2}$. Then, by an immediate verification, $\Gamma_{0} / H_{0}$ is isomorphic to the direct product of a cyclic group of order 2 and a dihedral group of order 8 .

We claim that $H_{0}$ is free of rank 3 . Let $L$ be the normal subgroup of $\Gamma_{0}$ generated by $a b$ : then $L$ has index 4 in $\Gamma_{0}$, contains $H_{0}$, and is shown, in the proof of $[\mathrm{GH}$, Lemma 11], to be isomorphic to $\mathbf{Z} *(\mathbf{Z} / 2 \mathbf{Z})$. 
Accordingly, by Kurosh's Theorem, if $H_{0}$ were not free, then it would contain a conjugate of $a b$, but this is not the case. Actually $H_{0}$ is contained in a subgroup of index 8 in $\Gamma_{0}$, free of rank 2 (see the proof of [GH, Lemma 11]), hence has rank 3.

Now, for $n \geq 1$, let us suppose that $\Gamma_{n-1}$ has a normal subgroup $H_{n-1}$ of index $2^{\alpha_{n-1}}$, which embeds as a subgroup of index $2^{\beta_{n-1}}$ in a direct product of $2^{n-1}$ nonabelian free groups of rank 3 .

The homomorphism $i_{n}$ described above embeds $\Xi_{n}$ as a subgroup of index 8 in $\Gamma_{n-1} \times \Gamma_{n-1}$. Define $H_{n}^{\prime}=i_{n}^{-1}\left(H_{n-1} \times H_{n-1}\right)$ : this a normal subgroup of index $2^{k}$ in $\Xi_{n}$, with $k \leq 2 \alpha_{n-1}$; so $H_{n}^{\prime}$ has index $2^{k+1}$ in $\Gamma_{n}$.

Then, using Lemmal7 $H_{n}=H_{n}^{\prime} \cap{ }^{a} H_{n}^{\prime}$ has index $2^{\alpha_{n}}$ in $G$, for some $\alpha_{n} \leq 4 \alpha_{n-1}+1$. Combining the inclusions $H_{n} \subset H_{n}^{\prime} \stackrel{\sim}{\rightarrow} H_{n-1} \times H_{n-1} \subset F_{3}^{2^{n-1}} \times F_{3}^{2^{n-1}}$, we obtain that $H_{n}$ embeds as a subgroup of index $2^{\beta_{n}}$ in ${F_{3}}^{2^{n}}$, with $\beta_{n}=\left[H_{n}^{\prime}: H_{n}\right]+2\left[F_{3}{ }^{2^{n-1}}\right.$ : $\left.H_{n-1}\right] \leq 2 \alpha_{n-1}+2 \beta_{n-1}$.

Define $\alpha_{n}^{\prime}=\left(13 \cdot 4^{n}-1\right) / 3$ and $\beta_{n}^{\prime}=\left(13 \cdot 4^{n}-15 \cdot 2^{n}+2\right) / 3$. Then $\alpha_{0}^{\prime}=4, \beta_{0}^{\prime}=0$, and they satisfy, for all $n$ : $\alpha_{n}^{\prime}=4 \alpha_{n-1}^{\prime}+1$ and $\beta_{n}^{\prime}=2 \alpha_{n-1}^{\prime}+2 \beta_{n-1}^{\prime}$. Therefore, an immediate induction gives $\alpha_{n} \leq \alpha_{n}^{\prime}$ and $\beta_{n} \leq \beta_{n}^{\prime}$ for all $n \geq 0$.

It is maybe worthwhile to restate the result avoiding reference to the particular sequence $\Gamma_{n}$.

Corollary 9. For every finitely presented group $G$ mapping onto the first Grigorchuk group $\Gamma$, there exist normal subgroups $N \subsetneq H$ in $G$, with $H$ of finite 2-power index, such that $H / N$ is isomorphic to a finite index subgroup in a direct product of nonabelian free groups.

Proof. Let $p: G \rightarrow \Gamma$ be onto. Since $G$ is finitely presented, $p$ factors through $\Gamma_{n}$ for some sufficiently large $n$, so that there exists a map $p^{\prime}$ of $G$ onto $\Gamma_{n}$. Then take $N=\operatorname{Ker}\left(p^{\prime}\right)$ and $H=p^{\prime-1}\left(H_{n}\right)$.

Combining Propositions 4 and 5, we also obtain the following statement:

Corollary 10. In the group $\Gamma_{i(n)}$, the $n$-ball consists of $2^{i(n)+1}$-torsion elements, and there exists a normal subgroup of index $2^{\alpha_{i(n)}}$, which embeds in a direct product of free groups, with $\alpha_{i(n)} \leq\left(13 \cdot n^{\log _{\lambda}(4)}-1\right) / 3$, and $\log _{\lambda}(4) \cong 6.60$.

Proof of Proposition 1, By Proposition 4, we may take $i$ sufficiently large so that the $n$-ball of $\Gamma_{i}$ consists of torsion elements. Since $H_{i}$ is a finite index subgroup in a nontrivial direct product of free groups (see Proposition [5), it has infinite abelianization. There is a short exact sequence

$$
1 \rightarrow H_{i} /\left[H_{i}, H_{i}\right] \rightarrow \Gamma_{i} /\left[H_{i}, H_{i}\right] \rightarrow \Gamma_{i} / H_{i} \rightarrow 1 .
$$

Accordingly, $G=\Gamma_{i} /\left[H_{i}, H_{i}\right]$ is an infinite, virtually abelian group, in which the $n$-ball consists of torsion elements. Moreover, since $\Gamma_{i} / H_{i}$ is a finite 2 -group, $G$ is also solvable. This proves (i).

For (iii), take, instead, $G=\Gamma_{i} /\left[\left[H_{i}, H_{i}\right],\left[H_{i}, H_{i}\right]\right]$. Since $H_{i}$ maps onto a nonabelian free group, its metabelianization is not virtually polycyclic, so that $G$ is virtually metabelian, but not virtually polycyclic.

For (ii), take a morphism of $H_{i}$ onto a polycyclic group $W$ which is not virtually nilpotent, and let $K$ be the kernel of this morphism. Since the normalizer of $K$ has finite index in $\Gamma_{i}, K$ has finitely many conjugates $K_{1}, \ldots, K_{\ell}$. Set $L=\bigcap_{j=1}^{\ell} K_{i}$. Then the diagonal map $H_{i} / L \rightarrow \prod_{j=1}^{\ell} H_{i} / K_{i}$ is injective, hence embeds $H_{i} / L$ in 
$W^{\ell}$. On the other hand, observe that $H_{i} / L$ projects onto $W$, so is not virtually nilpotent. It follows that $G=\Gamma_{i} / L$ is polycyclic but not virtually nilpotent. If $W$ has been chosen metabelian, then we also have that $G$ is virtually metabelian.

Proof of Proposition 3. Keep the last construction in the previous proof. Then $h\left(\Gamma_{i} / L\right) \leq h\left(\Gamma_{i}\right)$. Moreover, $h\left(\Gamma_{i} / L\right)>0$ since $\Gamma_{i} / L$ is solvable but not virtually nilpotent Os1. On the other hand, it is proved in GH] that $h\left(\Gamma_{i}\right) \rightarrow 0$. Thus we can obtain $h(G)$ arbitrarily small.

Remark 11. Consider for every $i$ an infinite quotient $Q_{i}$ of $\Gamma_{i}$. In the topology of marked groups (defined in [G2]; see also, for instance, [C]), the sequence $\left(Q_{i}\right)$ converges to the Grigorchuk group $\Gamma$. Indeed, otherwise by compactness it would have another cluster point, which would be a proper quotient of $\Gamma$, and therefore would be finite. This is a contradiction since the infinite groups form a closed subset in the topology of marked groups.

\section{APPENDIX}

We gather here the technical results concerning torsion in the groups $\Gamma_{n}$. They are slight modifications of results in the papers [GH, B1].

Recall that $\lambda \cong 1.25$ denotes the real root of the polynomial $2 X^{3}-X^{2}-X-1$. We introduce on $\Gamma_{-1}$ (hence on all its quotients) the metric $|\cdot|$ of [B1]: it is defined by attributing a suitable weight to each of the generators $a, b, c, d:|a|=2(\lambda-1) \cong 0.47$, $|b|=1-|a|=\lambda^{-3} \cong 0.53,|c|=2 \lambda^{2}-3 \lambda+1 \cong 0.34$, and $|d|=-2 \lambda^{2}+\lambda+2 \cong 0.19$. Throughout this appendix, unless explicitly stated, the balls and the lengths are meant in the sense of this weighted metric.

To check that the length of $a, b, c, d$ is exactly the weight we have imposed, it suffices to check this in the abelianization of $\Gamma_{-1}$, the $\mathbf{F}_{2}$-vector space with basis $(a, b, d)$ (which is also the abelianization of all $\left.\Gamma_{n}\right)$. There, it is a straightforward verification that the mapping $|\cdot|$ just defined extends to a length function by setting $|a \xi|=|a|+|\xi|$ for all $\xi \in\{b, c, d\}$.

Observe that if $\xi \in\{b, c, d\}$, and $i_{n}(\xi)=\left(\xi_{0}, \xi_{1}\right)$, we have

$$
\left|\xi_{0}\right|+\left|\xi_{1}\right|=\lambda^{-1}(|\xi|+|a|) \text {. }
$$

Lemma 12. Let $x \in \Gamma_{0}$ be any element. Set $x^{\prime}=x$ if $x \in \Xi_{0}$ and $x^{\prime}=x a$ otherwise; and set $i_{0}\left(x^{\prime}\right)=\left(x_{0}, x_{1}\right)$.

Then $\left|x_{0}\right|+\left|x_{1}\right| \leq \lambda^{-1}(|x|+|a|)$.

Suppose moreover that $x$ is of minimal length among its conjugates, and that $x \notin\{b, c, d\}$. Then $\left|x_{0}\right|+\left|x_{1}\right| \leq \lambda^{-1}|x|$.

Proof. Fix $x \in \Gamma_{0}$, and let $w$ be a word in the letters $\{a, b, c, d\}$, of minimal length ${ }^{1}$, representing $x$. Since every element in $\{b, c, d\}$ is the product of the two others, $w$ can be chosen so that no two consecutive letters are in $\{b, c, d\}$.

Suppose now that $x$ is of minimal length within its conjugacy class and that $w$ is not a single letter. Maybe conjugating $x$ by the last letter of $w$, we can suppose that $w$ ends with the letter $a$. The minimality assumption then implies that $w$ begins with a letter in $\{b, c, d\}$.

\footnotetext{
${ }^{1}$ If $w=u_{1} \ldots u_{n}$, the length of $w$ is defined as $\left|u_{1}\right|+\cdots+\left|u_{n}\right|$.
} 
First case: $x \in \Xi_{0}$. Write $w=\xi^{1}\left(a \xi^{2} a\right) \ldots \xi^{2 n-1}\left(a \xi^{2 n} a\right)$, where $\xi^{i} \in\{b, c, d\}$ for $i=1, \ldots, 2 n$. Write $i(x)=\left(x_{0}, x_{1}\right)$ and $i\left(\xi^{i}\right)=\left(\xi_{0}^{i}, \xi_{1}^{i}\right)$, so that $i\left(\xi^{i}\right)=$ $\left(\xi_{1}^{i}, \xi_{0}^{i}\right)$. Then

$$
\begin{aligned}
\left|x_{0}\right|+\left|x_{1}\right| & \leq\left(\left|\xi_{0}^{1}\right|+\left|\xi_{1}^{2}\right|+\cdots+\left|\xi_{0}^{2 n-1}\right|+\left|\xi_{1}^{2 n}\right|\right)+\left(\left|\xi_{1}^{1}\right|+\left|\xi_{0}^{2}\right|+\cdots+\left|\xi_{1}^{2 n-1}\right|+\left|\xi_{0}^{2 n}\right|\right) \\
& =\left(\left|\xi_{0}^{1}\right|+\left|\xi_{1}^{1}\right|\right)+\left(\left|\xi_{0}^{2}\right|+\left|\xi_{1}^{2}\right|\right)+\cdots+\left(\left|\xi_{0}^{2 n}\right|+\left|\xi_{1}^{2 n}\right|\right) .
\end{aligned}
$$

By (11), we get

$$
\left|x_{0}\right|+\left|x_{1}\right| \leq \lambda^{-1} \sum_{i=1}^{2 n}\left(\left|\xi^{i}\right|+|a|\right)
$$

On the other hand, $|x|=\sum_{i=1}^{2 n}\left(\left|\xi^{i}\right|+|a|\right)$, so that finally $\left|x_{0}\right|+\left|x_{1}\right| \leq \lambda^{-1}|x|$. Second case: $x \notin \Xi_{0}$, so that $x a \in \Xi_{0}$. Write $w=\xi^{1}\left(a \xi^{2} a\right) \ldots \xi^{2 n-1}\left(a \xi^{2 n} a\right) \xi^{2 n+1} a$, so that $\xi^{1}\left(a \xi^{2} a\right) \ldots \xi^{2 n-1}\left(a \xi^{2 n} a\right) \xi^{2 n+1}$ represents $x a$ in $\Gamma_{0}$. Write $i_{0}(x a)=$ $\left(x_{0}, x_{1}\right)$, and $i_{0}\left(\xi^{i}\right)=\left(\xi_{0}^{i}, \xi_{1}^{i}\right)$. Then

$$
\begin{aligned}
\left|x_{0}\right|+\left|x_{1}\right| \leq & \left(\left|\xi_{0}^{1}\right|+\left|\xi_{1}^{2}\right|+\cdots+\left|\xi_{0}^{2 n-1}\right|+\left|\xi_{1}^{2 n}\right|+\left|\xi_{0}^{2 n+1}\right|\right) \\
& +\left(\left|\xi_{1}^{1}\right|+\left|\xi_{0}^{2}\right|+\cdots+\left|\xi_{1}^{2 n-1}\right|+\left|\xi_{0}^{2 n}\right|+\left|\xi_{1}^{2 n+1}\right|\right) \\
= & \left(\left|\xi_{0}^{1}\right|+\left|\xi_{1}^{1}\right|\right)+\left(\left|\xi_{0}^{2}\right|+\left|\xi_{1}^{2}\right|\right)+\cdots+\left(\left|\xi_{0}^{2 n+1}\right|+\left|\xi_{1}^{2 n+1}\right|\right) \\
= & \lambda^{-1}\left(\sum_{i=1}^{2 n+1}\left(\left|\xi^{i}\right|+|a|\right)\right) \quad \text { again by (10). }
\end{aligned}
$$

Since $|x|=\sum_{i=1}^{2 n+1}\left(\left|\xi^{i}\right|+|a|\right)$, we get $\left|x_{0}\right|+\left|x_{1}\right| \leq \lambda^{-1}|x|$.

The other inequality $\left|x_{0}\right|+\left|x_{1}\right| \leq \lambda^{-1}(|x|+|a|)$ is proved similarly: we must deal with the following cases:

- $w$ begins and ends with the letter $a$ : considering whether or not $x \in \Xi_{0}$, in both cases we obtain the stronger inequality $\left|x_{0}\right|+\left|x_{1}\right| \leq \lambda^{-1}(|x|-|a|)$.

- $w$ begins and ends with letters in $\{b, c, d\}$ : considering whether or not $x \in \Xi_{0}$, in both cases we obtain the inequality $\left|x_{0}\right|+\left|x_{1}\right| \leq \lambda^{-1}(|x|+|a|)$.

- $w$ begins with the letter $a$ and ends with a letter in $\{b, c, d\}$ : in this case, replacing $x$ by $x^{-1}$ and $w$ by $w^{-1}$ (this is the word $w$ read from right to left - recall that the generators are involutions), we reduce to the case, already carried out, in which $w$ begins with a letter in $\{b, c, d\}$ and ends with the letter $a$, obtaining the inequality $\left|x_{0}\right|+\left|x_{1}\right| \leq \lambda^{-1}|x|$.

Since the verifications are similar to the computations above and since we do not use this case in the sequel, we omit the details.

Lemma 13. For every $n \geq-1$, and every element in the open $\lambda^{n-1}$-ball of $\Gamma_{-1}$, its image in $\Gamma_{n}$ is of $2^{n+1}$-torsion.

Proof. For $n=-1, \lambda^{-2}=|a|+|d| \cong 0.66$, and the elements in the open $\lambda^{-2}$-ball are $1, a, b, c$, and $d$, and are of 2-torsion in $\Gamma_{-1}$.

For $n=0, \lambda^{-1}=|a|+|c| \cong 0.81$, and the elements in the open $\lambda^{-1}$-ball are, besides the elements in the open $\lambda^{-2}$-ball already quoted, ad and its inverse $d a$, which are of 4 -torsion in $\Gamma_{0}$. 
We can start an induction, and suppose that, for some $n \geq 1$, we have already proved that for every element in the open $\lambda^{n-2}$-ball of $\Gamma_{-1}$, its image in $\Gamma_{n-1}$ is of $2^{n}$-torsion. Pick $x$ in the open $\lambda^{n-1}$-ball of $\Gamma_{-1}$. We want to show that $x^{2^{n+1}}=1$. We can suppose that $x$ is of minimal length among its conjugates, and that $x \notin\{b, c, d\}$. Define $x^{\prime}$ as in Lemma 12, i.e. $\left\{x^{\prime}\right\}=\{x, x a\} \cap \Xi_{-1}$. Denote by [.] the projection of $\Gamma_{-1}$ onto $\Gamma_{0}$. Set $i_{0}\left(\left[x^{\prime}\right]\right)=\left(x_{0}, x_{1}\right)$.

First case: $x \in \Xi_{-1}$, i.e. $x=x^{\prime}$. By Lemma 12, for $i=0,1,\left|x_{i}\right| \leq\left|x_{0}\right|+\left|x_{1}\right| \leq$ $\lambda^{-1}|x| \leq \lambda^{n-2}$. By the induction hypothesis, $x_{0}$ and $x_{1}$ are of $2^{n}$-torsion in $\Gamma_{n-1}$. Since $i_{0}$ induces an injection of $\Xi_{n}$ into $\Gamma_{n-1} \times \Gamma_{n-1}$, this implies that $x$ is of $2^{n}$-torsion, hence of $2^{n+1}$-torsion in $\Gamma_{n}$.

Second case: $x \notin \Xi_{-1}$, i.e. $x^{\prime}=x a$. Then $x^{2} \in \Xi_{-1}$, and

$$
i_{0}\left(\left[x^{2}\right]\right)=i_{0}([x a a x a a])=i_{0}([x a]) i_{0}([a(x a) a])=\left(x_{0} x_{1}, x_{1} x_{0}\right),
$$

which is conjugate to $\left(x_{0} x_{1}, x_{0} x_{1}\right)$ in $\Gamma_{-1} \times \Gamma_{-1}$. By Lemma 12, we have $\left|x_{0} x_{1}\right| \leq\left|x_{0}\right|+\left|x_{1}\right| \leq \lambda^{-1}|x| \leq \lambda^{n-2}$. By the induction hypothesis, $x_{0} x_{1}$ is of $2^{n}$-torsion in $\Gamma_{n-1}$, so $i_{0}\left(\left[x^{2}\right]\right)$ is of $2^{n}$-torsion in $\Gamma_{n-1} \times \Gamma_{n-1}$. Since $i_{0}$ induces an injection of $\Xi_{n}$ into $\Gamma_{n-1} \times \Gamma_{n-1}$, this implies that $x^{2}$ is of $2^{n}$-torsion in $\Xi_{n} \subset \Gamma_{n}$, hence $x$ is of $2^{n+1}$-torsion in $\Gamma_{n}$.

Proof of Proposition 4. Suppose that $x$ has word length $\leq n$. Then

$$
|x| \leq|b| n=\lambda^{-3} n=\lambda^{\log _{\lambda}(n)-3}<\lambda^{i(n)-1} .
$$

By Lemma 13, the image of $x$ in $\Gamma_{i(n)}$ is of $2^{i(n)+1}$-torsion.

Acknowledgement. We thank Emmanuel Breuillard for encouragement and valuable discussions. We also thank Goulnara Arzhantseva and Pierre de la Harpe for useful remarks.

\section{REFERENCES}

[B1] Laurent BARTHOLDI. The growth of Grigorchuk's torsion group. Int. Math. Res. Not. 20, 1049-1054 (1998).

[B2] Laurent BARThOLDI. A Wilson group of non-uniformly exponential growth. C. R. Math. Acad. Sci. Paris 336, no.7, 549-554 (2003).

[C] Christophe Champetier. L'espace des groupes de type fini, Topology 39, no.4 657-680 (2000).

[G1] Rostislav GRigorchuk. On the Burnside problem for periodic groups. Funct. Anal. Appl. 14, 41-43, 1980 (Russian original: Funktsional. Anal. i Prilozhen 14, 53-54, 1980).

[G2] Rostislav I. Grigorchuk. Degrees of growth of finitely generated groups, and the theory of invariant means. Math. USSR Izv. 25:2, 259-300, 1985 (Russian original: Izv. Akad Nauk. SSSR Ser. Mat. 48, no.5, 939-985, 1984).

[GH] Rostislav I. GRIGORChUK, Pierre DE LA HARPE. Limit behaviour of exponential growth rates for finitely generated groups. Monogr. Enseign. Math. 38, 351-370 (2001).

[H] Pierre DE LA HARPE. "Topics in geometric group theory". University of Chicago Press, Chicago, IL, 2000.

[L] Igor G. Lysionok. A set of defining relations for the Grigorchuk group. Math. Notes Acad. Sc. USSR 38:4, 784-792, 1985 (Russian original: Mat. Zametki 38, 503-511, 1985).

[Ol] Alexander OlshanskII. An infinite group with subgroups of prime orders. Math. USSR Izv. 16, 279-289, 1981 (Russian original: Izv. Akad. Nauk SSSR Ser. Mat. 44, 309-321, 1980). 
[Os1] Denis V. Osin. The entropy of solvable groups. Ergod. Theory \& Dynam. Sys. 23, no.3, 907-918 (2003).

[Os2] Denis V. Osin. Algebraic entropy of elementary amenable groups. Preprint 2005, to appear in Geom. Dedicata.

[Se] Jean-Pierre SERre. "Arbres, amalgames, SL 2 ". Astérisque 46, SMF, 1977.

[St] John R. Stallings. On torsion-free groups with infinitely many ends. Ann. of Math. 88, 312-334 (1968).

[W] John S. WiLsOn. On exponential growth and uniformly exponential growth for groups. Invent. Math. 155, 287-303 (2004).

Laurent Bartholdi

E-mail: laurent.bartholdi@epfl.ch

École Polytechnique Fédérale de Lausanne (EPFL)

Institut de Mathématiques B (IMB)

CH-1015 Lausanne, Switzerland

Yves de Cornulier

E-mail: decornul@clipper.ens.fr

Université de Neuchâtel

Institut de Mathématiques

Rue Émile Argand 11

CH-2007 Neuchâtel, Switzerland 